\title{
Synthesis and Characterization of Iron Oxide Nanoparticles with Enhanced Magnetization Using Pluronic F-127
}

Frederico Gutierrez ${ }^{1}$, Jefferson Araujo ${ }^{1}$, Geronimo Perez ${ }^{2}$, Jules Gardener ${ }^{3}$ and Guillermo Solórzano ${ }^{4}$

${ }^{1}$ Department of Physics, Pontifical Catholic University of Rio de Janeiro-Rio, Rio de Janeiro, Rio de Janeiro, Brazil, ${ }^{2}$ Materials Metrology Division, National Institute of Metrology, Standardization and Industrial Quality (Inmetro), Rio de Janeiro, Rio de Janeiro, Brazil, ${ }^{3}$ Harvard University Center for Nanoscale Systems, Cambridge, Massachusetts, United States, ${ }^{4}$ Department of Chemical and Materials Engineering, Pontifical Catholic University of Rio de Janeiro, Rio de Janeiro, Rio de Janeiro, Brazil

Magnetic nanoparticles (MNPs) have been highly useful for the advancement of areas such as science and technology, standing out in biology and medicine research. In some methods such as bioseparation, drug delivery, magnetic particle imaging (MPI), and cancer hyperthermia [1], for better use of MNPs in most medical applications, the core size must be around 10-50 $\mathrm{nm}$. Moreover, these particles maintain their magnetic properties, which depend on the composition, size distribution and degree of crystallinity. Thus, the MNPs obtained in this study were produced by co-precipitation, which has been shown to be the best method for the production of MNPs, due to the amount of monodispersed sample produced [1-2]. The reagents ferric chloride hexahydrate $\left(\mathrm{FeCl}_{3} .6 \mathrm{H}_{2} \mathrm{O}\right)$, ferrous chloride tetrahydrate $\left(\mathrm{FeCl}_{2} .4 \mathrm{H}_{2} \mathrm{O}\right)$, ammonium hydroxide $\left(\mathrm{NH}_{4} \mathrm{OH}, 30 \%\right)$, hydrochloride acid $(\mathrm{HCl}, 38 \%)$, and Pluronic-F127 were used in the synthesis of three different samples (Pluronic 0.200, Pluronic 0.400, and MNPs), which were prepared using iron salt mixture as follow: Two iron salt solutions were prepared, one by dissolving $6.8 \mathrm{~g}$ of $\mathrm{FeCl}_{3} .6 \mathrm{H}_{2} \mathrm{O}$ in $25 \mathrm{~mL}$ of distilled water and another by dissolving $3.95 \mathrm{~g}$ of $\mathrm{FeCl}_{2} .4 \mathrm{H}_{2} \mathrm{O}$ in $10 \mathrm{~mL}$ of aqueous $\mathrm{HCl}$ solution $(5.49 \mathrm{M})$; subsequently, they were mixed together in a proportion of $4 \mathrm{~mL}$ of iron (III) to $1 \mathrm{~mL}$ of iron (II). Pluronic 0.200 and Pluronic 0.400 are samples coated with Pluronic-F127. The first one it was obtained from the addition of an 8-mL iron mixture to an aqueous solution containing 50 $\mathrm{mL}$ of $\mathrm{NH}_{4} \mathrm{OH}(1.30 \mathrm{M})$ and $10 \mathrm{~mL}$ of Pluronic-F127 $(0.30 \mathrm{M})$. The second sample was also prepared using $8 \mathrm{~mL}$ of iron mixture; however, it was added to a solution with a higher concentration of PluronicF127 (0.60 M). The MNPs was the pure sample, obtained by adding the iron salt mixture to the aqueous $\mathrm{NH}_{4} \mathrm{OH}$ solution $(1.30 \mathrm{M})$. The three basic solutions were previously heated to $80^{\circ} \mathrm{C}$ for 10 minutes, with vigorous stirring. At the end of the process, the three samples were kept in an ultrasonic bath for 1 hour to avoid agglomeration [3]. The size distribution and the magnetic behavior of the nanoparticles were studied by transmission electron microscopy (TEM), dynamic light scattering (DLS), and scanning magnetic microscopy (SMM). The average diameter of MNPs (around $11 \mathrm{~nm}$ ), Pluronic 0.200 (around 10 $\mathrm{nm}$ ) and Pluronic 0.400 (around $6 \mathrm{~nm}$ ) was estimated based on the magnetization curve [3]. The average diameter of MNPs are usually obtained by using techniques such as TEM (See FIG. 1.a and 1.b), which, in our study, obtained an average diameter of $12 \mathrm{~nm}$ (FIG. 1.a) containing a histogram for the MNPs sample and $7 \mathrm{~nm}$ (Fig. 1.b) for the pluronic 0.400. The particle size distribution of the magnetic nanoparticles was also estimated by DLS. The equipment was a Horiba model Nanopartica SZ-100 using a $10 \mathrm{~mW}$ laser with an incident beam with a wavelength of $532 \mathrm{~nm}$. The MNPs sample has a diameter of $207 \mathrm{~nm}$, pluronic 0.200 has a 460nm and pluronic 0.400 has a $185 \mathrm{~nm}$ (See FIG. 1.c). The nanoparticle diameter obtained by DLS was larger than that using the other techniques because the DLS technique measures the hydrodynamic diameter of the particles in suspension. It's important to emphasize through the data obtained by techniques, that the increase in the concentration of pluronic F-127 the size of the MNPs decrease. The coated samples were analised using a JEOL ARM instrument operating in TEM and 
STEM modes under 200kV. Both analyzed samples, pluronic 0.200 and pluronic 0.400 , exhibit a typical electron diffraction pattern (EDP) of the magnetite structure. Fig.2a and Fig.2d show the indexed EDPs of these both samples respectively with highlighted rings help visualization. In the TEM images of the Fig.2b and Fig.2e it is possible to compare the size of the samples pluronic 0.200 and pluronic 0.400 respectively. The particles of the sample pluronic 0.200 show smaller size than the particles of the sample pluronic 0.400. The high angle annular dark field (HAADF) image of Fig.2c shows several magnetite nanoparticles of faceted polygonal morphology, however, in the HAADF image of the Fig.2c is possible to observe less particles with polygonal morphology [4].
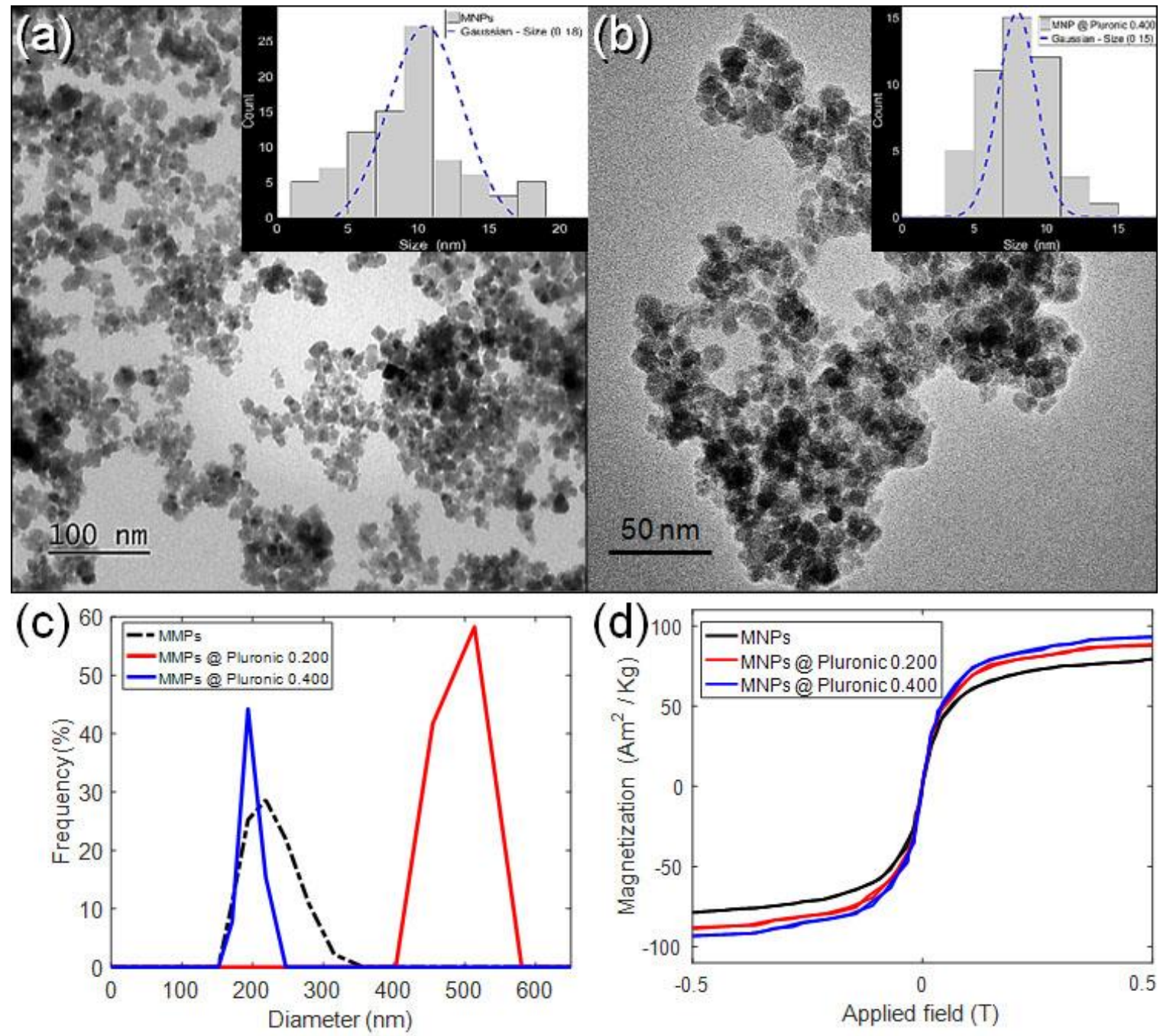

Figure 1. Bright field micrographs and respective average size in histograms of: (a) Sample MNPs and (b) sample Pluronic 0.400. (c) DLS curves of MNPs, Pluronic 0.200 and Pluronic 0.400. (d) Magnetization curves of MNPs, Pluronic 0.200 and Pluronic 0.400. 


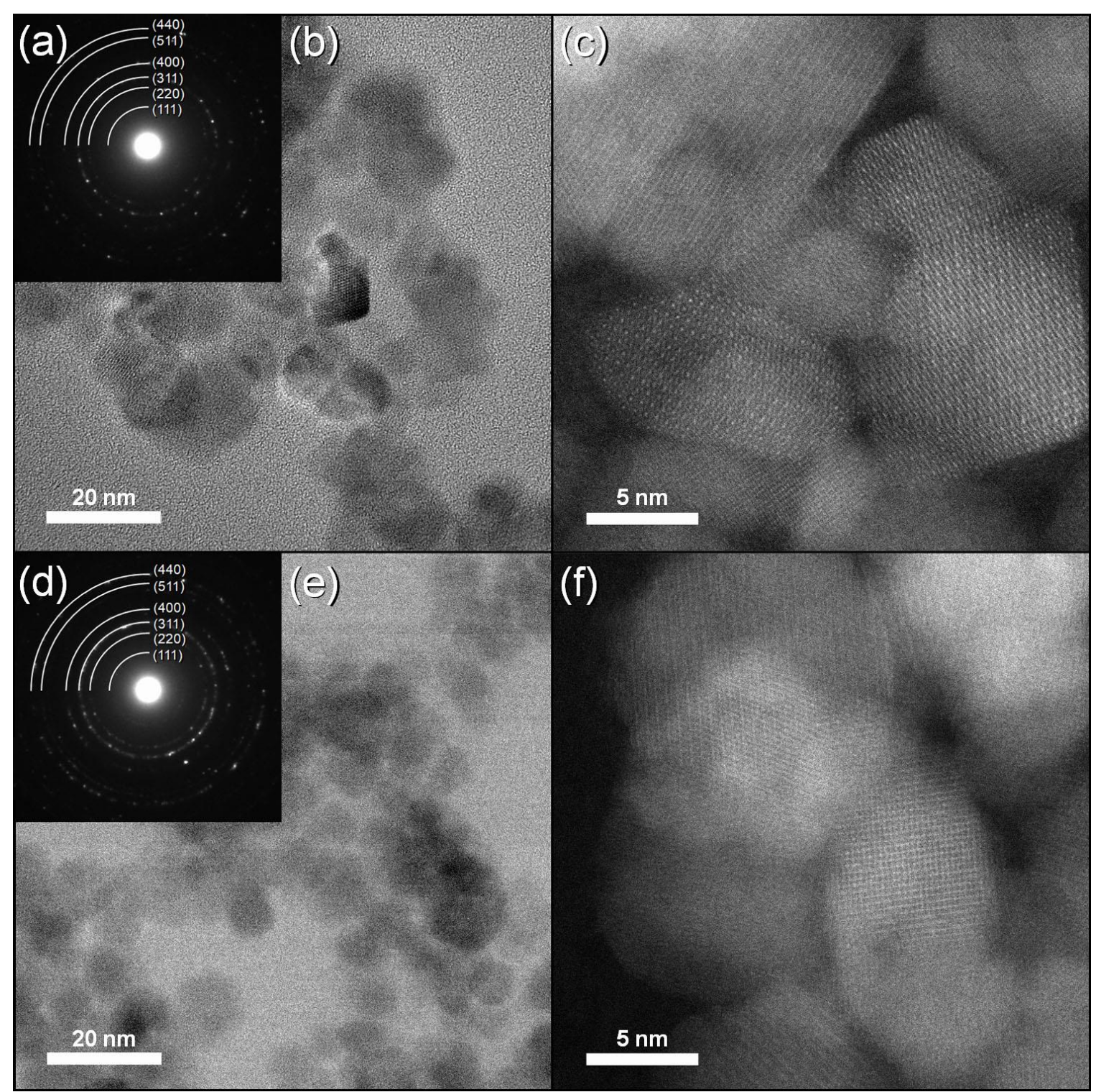

Figure 2. TEM images of both magnetite samples: (a) EDP of sample Pluronic 0.200. (b) TEM micrograph of sample Pluronic 0.200. (c) HAADF imaging of sample Pluronic 0.200. (d) EDP of sample Pluronic 0.400. (e) TEM micrograph of sample Pluronic 0.400. (f) HAADF imaging of sample Pluronic 0.400 .

\section{References}

1. D. Shi., Nanoscience in Biomedicine 1 (2009) Pag. 291 - 313.

2. S. Arsalni, ACS Sustainable Chem. Eng. 6 (2018) 1 - 11.

3. J. F. D. F. Araujo, Construção de um magnetômetro Hall a baixas temperaturas para caracterização de nanopartículas magnéticas. PUC (2013). Pag. 76-78.

4. Acknowledgments: CNPq (Brazil) and Capes (Brazil) for financial support. 